\title{
Expression of p-AKT characterizes adenoid cystic carcinomas of head and neck with a higher risk for tumor relapses
}

\author{
Hans-Ullrich Völker*1, Matthias Scheich ${ }^{2}$, Annette Berndt ${ }^{3}$, Imme Haubitz ${ }^{2}$, \\ Alexandra Metzger ${ }^{1}$, Hans-Konrad Müller-Hermelink ${ }^{1}$, Ulrike Kämmerer ${ }^{3}$ \\ and Melanie Schmidt ${ }^{3}$
}

\begin{abstract}
Address: ${ }^{1}$ Institute for Pathology, University Würzburg, Würzburg, Germany, ${ }^{2}$ Dept. of Otorhinolaryngology, University Würzburg, Würzburg, Germany and ${ }^{3}$ Dept. of Gynaecology, University Würzburg, Würzburg, Germany

Email: Hans-Ullrich Völker* - ullrich.voelker@mail.uni-wuerzburg.de; Matthias Scheich - matthias.scheich@gmx.de; Annette Berndt - diamonds.are.forever@web.de; Imme Haubitz - imme.haubitz@gmx.de; Alexandra Metzger - alexandra.metzger@gmx.de; Hans-Konrad Müller-Hermelink - path062@mail.uni-wuerzburg.de; Ulrike Kämmerer - u.kaemmerer@mail.uni-wuerzburg.de;

Melanie Schmidt - melanie_weigand@hotmail.de

* Corresponding author
\end{abstract}

Published: 19 June 2009

Diagnostic Pathology 2009, 4:18 doi:10.1186/1746-1596-4-18
Received: 20 April 2009

Accepted: 19 June 2009

This article is available from: http://www.diagnosticpathology.org/content/4/1/18

(C) 2009 Völker et al; licensee BioMed Central Ltd.

This is an Open Access article distributed under the terms of the Creative Commons Attribution License (http://creativecommons.org/licenses/by/2.0), which permits unrestricted use, distribution, and reproduction in any medium, provided the original work is properly cited.

\begin{abstract}
Background: Adenoid cystic carcinomas are rare tumors with an indolent clinical course, but frequent local relapses. The identification of tumors with a higher relapse risk seems to be interesting. Hence we investigated parameters of glucose metabolism, which were found associated with poor prognosis in other malignancies.
\end{abstract}

Methods: Specimen of 29 patients were investigated immunohistochemically with antibodies against p-AKT, TKTL-I (transketolase-like I), M2PK (M2 pyruvate kinase), and GLUT-I. Proliferation was investigated by staining with Ki67. The tumors were located at the major or minor salivary glands. Only the typical cribriform subtype was investigated. The initial tumor stage was PTI or PT2.

Results: Expression of $p-A K T$ was significantly $(P=0.036)$ associated with a higher relapse risk in multivariate analysis. Low expression of M2PK was non-significantly $(P=0.065)$ predictive for a higher risk. TKTL-I and GLUT-I were expressed in the majority of cases, albeit not associated with relapse risk.

Conclusion: Adenoid cystic carcinomas positive for p-AKT show a higher relapse risk. However, other parameters of glucose metabolism investigated here or proliferation (Ki67) were not predictive in this entity. Our findings demonstrate a possible background for therapeutic approaches targeting the inhibition of PI3K/AKT pathway.

\section{Background}

Malignant tumors of salivary glands represent a small subset of all malignancies. Adenoid cystic carcinomas (ACC) are one of the most common types at this anatomic site.
They account for $1 \%$ of all malignant head and neck tumors, $10 \%$ of all salivary gland tumors, and $22 \%$ of malignant salivary gland tumors $[1,2]$. The majority occures at the minor salivary glands $(60 \%)[3]$. From the 
major salivary glands, the parotid is most often involved. Locations like the nasal cavity and the paranasal sinus are also possible. Typically, ACC show an indolent clinical course with a considerable risk for local relapse $(60 \%$ of patients, mostly $<2$ years after primary) and late distant metastases (lung or bone), the latter sometimes several years after first diagnosis with fatal outcome. Standard treatment for ACC is surgery, followed by post-operative radiotherapy [4]. Since tumors show a characteristic perineural spread, the surgical key problem is the complete resection. Therefore, the extend of tumor infiltration is sometimes not apparent, and local relapses may originate from microscopic residual disease.

The therapeutic options have not been improved significantly in recent years despite different studies which investigated the influence of chemotherapy or molecular approaches $[3,5,6]$. However, there are individual differences concerning the outcome. To estimate prognosis, tumor stage, subtype, and the extend of perineural spread were found to be reliable.

In order to identify additional predictive parameters by immunohistochemical investigation, we performed the following study. The intention herein was to clarify the predictive potential of the expression of several markers which are assumed to play an important role in the glucose metabolism, especially phosphorylated AKT (pAKT), transketolase-like-1 (TKTL-1), M2 pyruvate kinase (M2PK), and glucose transporter (GLUT-1). Their expression was found associated with a poor prognosis in different malignancies [7-15]. To our knowledge, these parameters were not investigated in ACC up to now. The idea for this study is based on the theory of Otto Heinrich Warburg (1883-1970) from 1924, which implies the increase of glucose utilization in all malignant tumors [16].

\section{Methods}

Paraffin embedded tumor specimens of 29 patients (16 female, 13 male) with ACC were included in this study.

Table I: Site of primary tumors.

\begin{tabular}{ccc}
\hline Site & $\mathrm{n}=\mathbf{2 9}$ & $\%$ \\
\hline Glandula parotidea & 4 & 13.8 \\
\hline Glandula submandibularis & 5 & 17.2 \\
\hline Glandula sublingualis & 1 & 3.5 \\
\hline Minor salivary glands & 10 & 34.5 \\
\hline Nasal cavity and paranasal sinus & 9 & 31.0 \\
\hline
\end{tabular}

The locations of primaries are indicated in table 1 . In cases with relapses, primaries and their relapses were examined. The time of follow up observation was at least five years. The initial therapy was surgical with consecutive local radiation in all cases. Tumors investigated in this study had a initial tumor stage of pT1 or pT2, N0, MO.

Three histological subtypes of ACC exist (cribriform, solid, tubular). Due to the known differences in prognosis [2], only cases with the typical cribrifom pattern of growth and typical immunophenotyp (Vimentin +, CD117+) were included. Diagnoses were validated by two experienced pathologists.

\section{Immunohistochemical staining}

Immunohistochemical staining with commercially available antibodies were done in standard technique following manufacturer's protocols. Positive controls were used in accordance with manufacturer's recommendations or own established/published experiences. Antibodies including provider, dilution and positive controls are listed in table 2. All sections including positive controls were stained simultaneously for the appropriate antibody.

Slides with tumor sections with a maximum thickness of 2-5 $\mu \mathrm{m}$ were deparaffinized with xylene. Rinsing the slides in decreasing concentrations of ethanol was followed by antigen unmasking in $10 \mathrm{mM}$ sodium citrate buffer $(\mathrm{pH}=6.0)$ in a microwave oven at $600 \mathrm{~W}$ for $5 \mathrm{~min}$ utes. After rinsing in distilled $\mathrm{H}_{2} \mathrm{O}$, endogenous peroxidase was inhibited by incubation for 10 minutes in $3 \%$ $\mathrm{H}_{2} \mathrm{O}_{2}$ in methanol. Slides were then washed with PBS and incubated with 1\% goat serum in PBS for 15 minutes. Subsequently, slides were incubated with the respective primary antibodies diluted in antibody diluent (DAKO, Hamburg, Germany).

After 45-60 minutes of incubation at room temperature, slides were washed in PBS, incubated with an appropriate biotinylated secondary antibody (DAKO), washed and incubated with streptavidin-peroxidase (DAKO). Staining was visualized by adding 3.3'-diaminobenzidine (DAB; DAKO) with subsequent counterstaining using haematoxylin. Sections were dehydrated in graded ethanol and embedded in Vitro Clud (Langenbrinck, Germany).

\section{Statistical analysis and clinical correlation}

Staining intensities were scored independently by two observers with experiences in immunohistochemical investigations. Differences were discussed (no differences between negative or positive, but some between low and moderate staining intensity). Scoring grades were 0 (negative), 1 (low), 2 (moderate) and 3 (high staining intensity). Examples are shown in Figure 1. 
Table 2: Used antibodies, source and dilution.

\begin{tabular}{ccccc}
\hline Antibody & Clone/species & Source & Dilution & Positive control \\
\hline P-AKT & ab2882I polyclonal rabbit & Abcam, Cambridge, UK & $1: 100$ & glioblastoma multiforme \\
\hline TKTL-I & JFCI2TI0 monoclonal mouse & Linaris, Wertheim, Germany & $1: 200$ & colorectal adenocarcinoma \\
\hline M2PK & DF4 & ScheBo Biotech, Gießen, Germany & $1: 250$ & colorectal adenocarcinoma \\
\hline GLUT-I & polyclonal rabbit & DAKO, Hamburg, Germany & $1: 100$ & epidermis \\
\hline Ki67 & MIB-I & DAKO, Hamburg, Germany & I:200 & tonsil \\
\hline
\end{tabular}

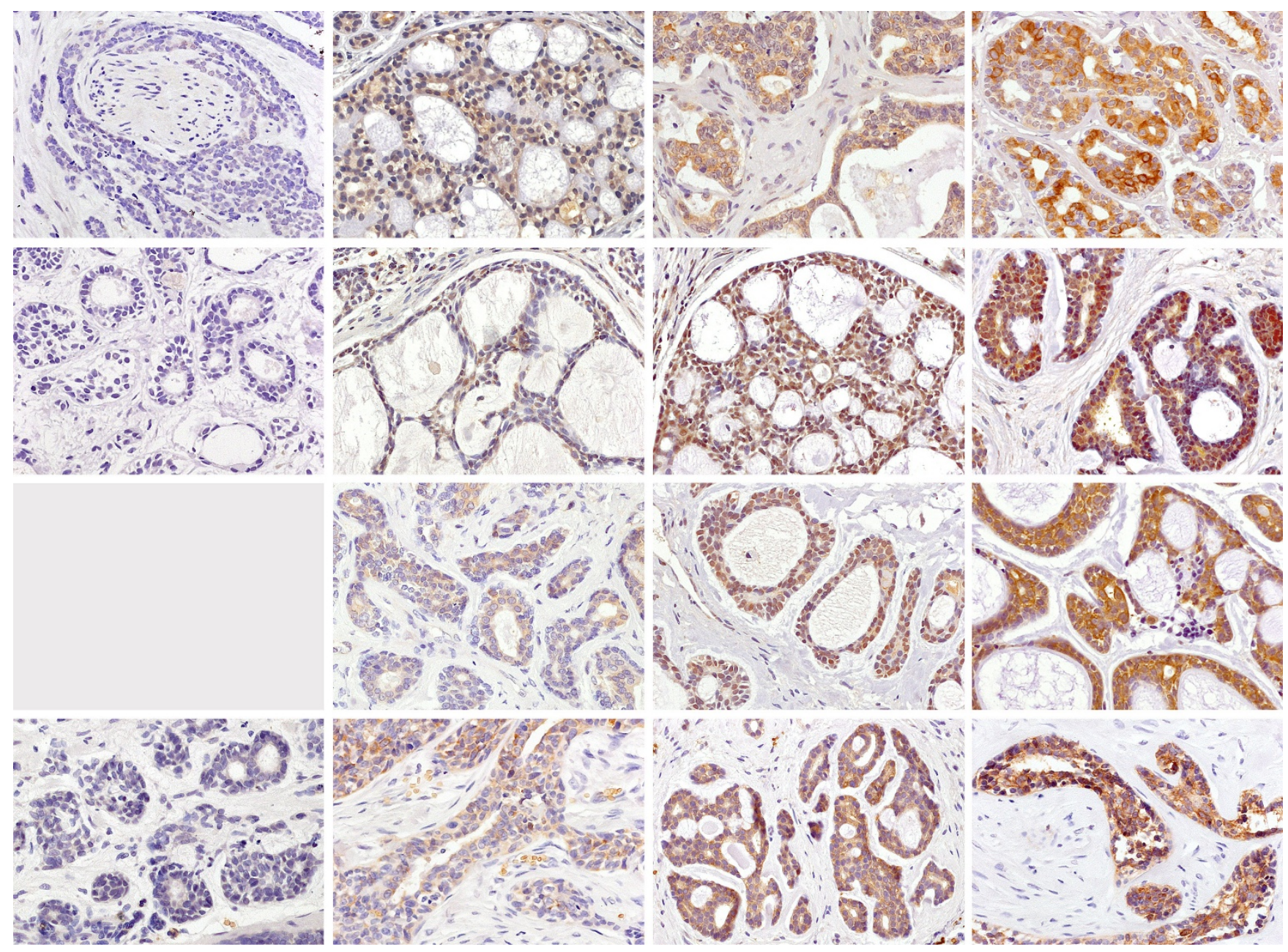

Figure I

Examples for immunohistochemical staining results: row I: P-AKT, row 2: TKTL- I, row 3: M2PK, row 4: GLUT-I; column I: negative (no negative case was found when M2PK was stained), column 2: weak, column 3: moderate, column 4: strong. 
Statistical tests were done using the SPSS software package (version 14.0.1, Chicago, IL). Charcteristics are presented at means with their SD values for normally distributed variables. The significance level was set at $\alpha=5 \%$ for all comparisons. Significances were assessed with log-rank statistics, Kendall rank correlation, U-Test by Mann and Whitney, or chi-square test. The cox proportional hazards model was used for the multivariate analysis.

\section{Ethics}

The ethic approval for this study was obtained from Ethic Committee of the Medical Faculty of University Wuerzburg. The study was performed in accordance to the Helsinki declaration.

\section{Results}

Patient's median age was 58 years $( \pm 15,32-86$ years $) .16 /$ $29(55.2 \%)$ of patients were free of relapses after initial surgery. The remaining patients developed relapses within 2-94 months after initial surgery (median 35 months). Figure 2 shows the overall relapsing course. If relapsing, 6 patients had more than one relapse (2-4). The site of primary and risk for relapse did not significantly correlate in this cohort $(P=0.1)$. However, 70\% (7/10) of tumors located in the major salivary glands were disease-free after initial therapy, whereas the majority of relapses arose from tumors of other sites (76.9\%). The tumor stage did not influence the risk for relapse in this study.
Comparing the staining results of primaries and relapses of each single patient, no significant differences were found ( $\mathrm{P}>0.8$, U-Test by Mann and Whitney). Therefore, in the following the overall staining results are reported.

The proliferation (Ki67) reached from $<10 \%$ up to $50 \%$ in primaries and relapses. The differences were not significant and did not show a trend to higher proliferation in relapsing cases. Figure 3 shows the results and distribution of immunohistochemical staining. Significant correlations of different staining results are given in table 3 . Interestingly, elder patients showed a significant lower expression of M2PK ( $\mathrm{tau}=-0.28, \mathrm{P}=0.031$, Kendall rank correlation). For the different sites, tumors of nasal cavity or paranasal sinuses stained weaker for TKTL-1 than others. Staining intensities were distributed equally in all tumors with $80-100 \%$ positive tumor cells. A focal staining was not observed.

The multivariate analysis for the predictive role of different factors regarding the relapse risk is indicated in table 4. Apart from well known parameters (status of complete surgical resection), expression of p-AKT was significantly $(P=0.036)$ associated with a higher relapse risk. Furthermore, lower expression of M2PK was a non-significant ( $\mathrm{P}$ $=0.065$ ) predictor for a higher relapse risk.

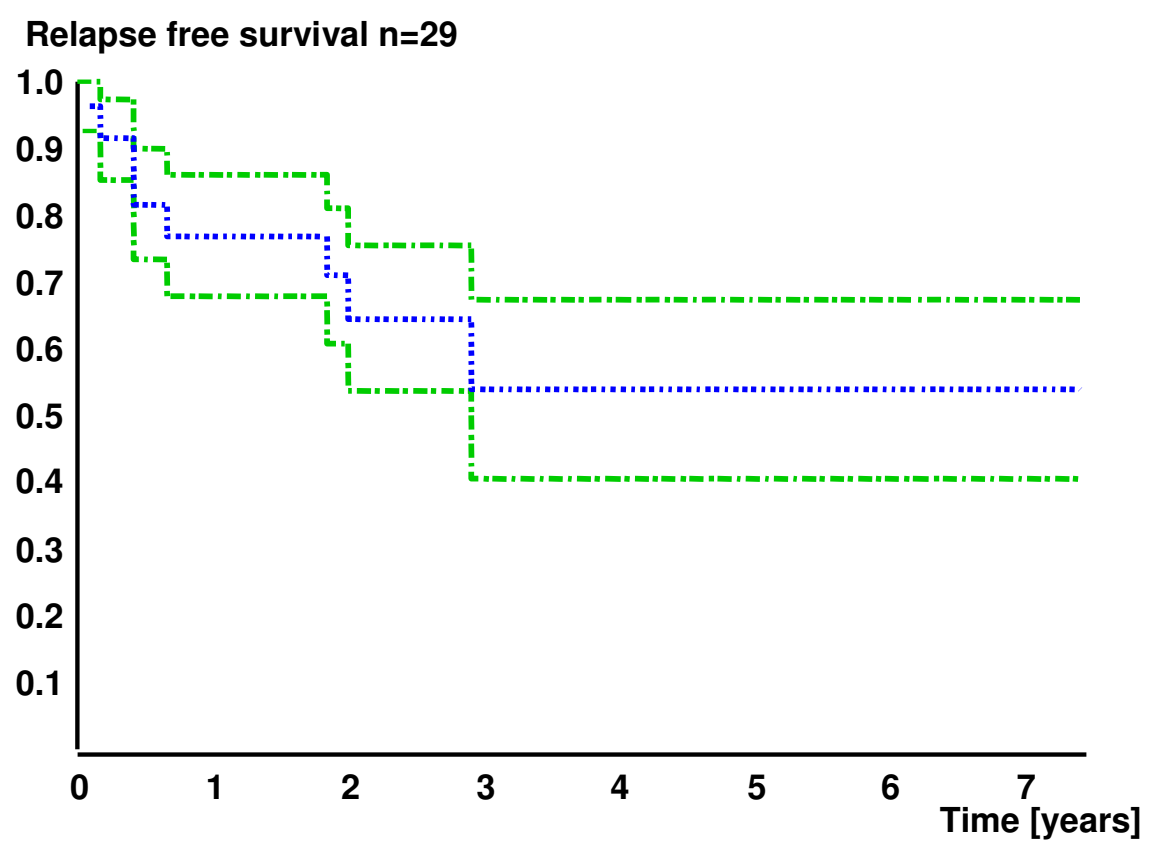

Figure 2

Relapsing course (overall) with $95 \%$ confidence interval. 


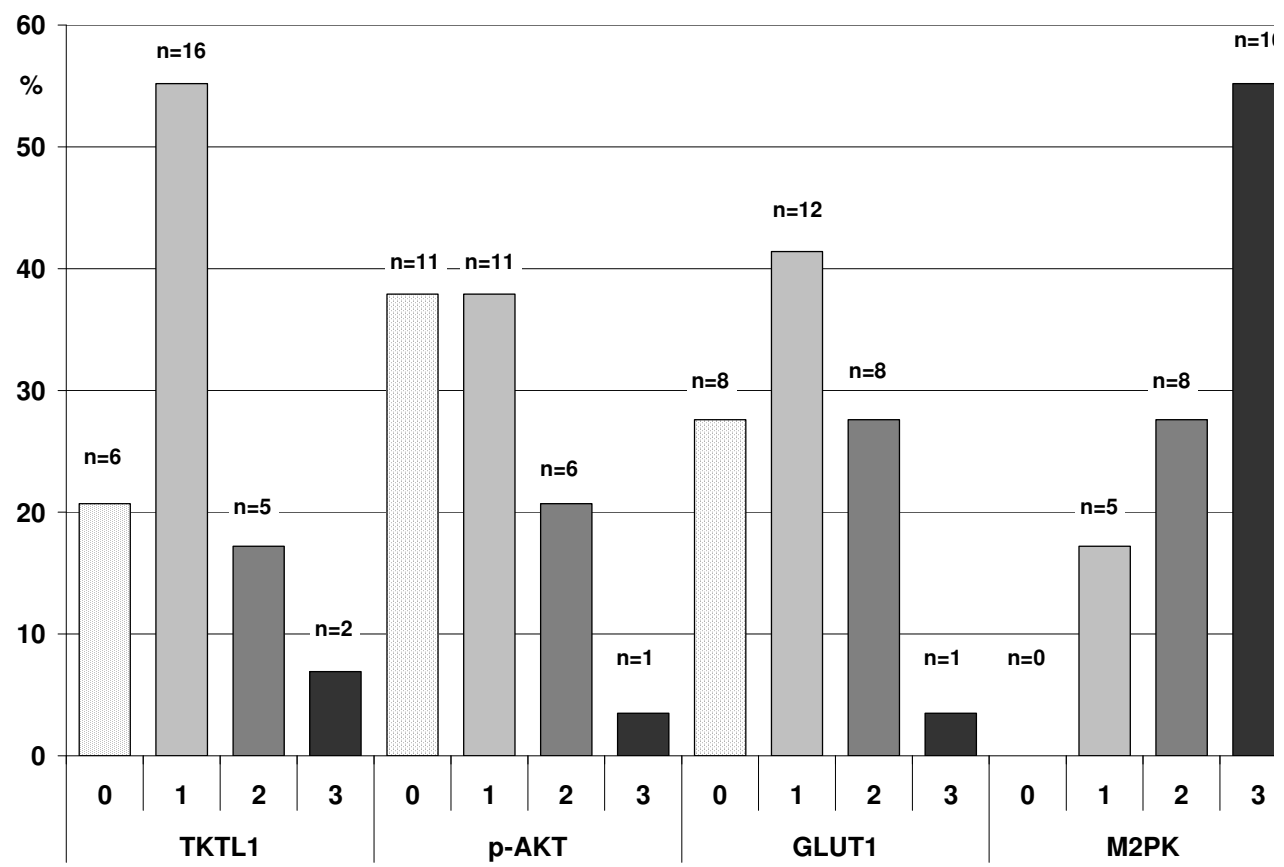

Figure 3

Results of immunohistochemical staining results in $\mathbf{2 9}$ adenoid cystic carcinomas. Staining intensity (Score): 0-negative, I-low expression, 2-moderate expression, 3-strong expression

\section{Discussion}

Since Otto Warburg has published his hypothesis of the importance of glucose metabolism in tumor cells, many studies have been published regarding this issue. The influence of PI3K-AKT pathway, glucose transporter GLUT-1 and M2 pyruvate kinase on glycolysis was demonstrated in several investigations $[11,12,15]$. In addition, transketolase-like-1 (TKTL-1) was postulated to play also an important role in the glucose metabolism of tumors [17]. To our knowledge, this is the first study which elucidates the role of the markers mentioned above in adenoid cystic carcinomas (ACC) of head and neck.

Table 3: Correlation between different staining results (Kendall rank correlation).

\begin{tabular}{cccc}
\hline \multicolumn{2}{c}{ Stainings } & tau & P \\
\hline P-Akt & TKTL-I & 0.3848 & 0.0034 \\
\hline P-Akt & M2PK & 0.4015 & 0.0022 \\
\hline TKTL-I & Glut-I & 0.2650 & 0.044 \\
\hline TKTL-I & M2PK & 0.5395 & $<0.00005$ \\
\hline
\end{tabular}

All markers were expressed in ACC of our cohort. In all tumors, $80-100 \%$ of tumor cells were positive stained. Therefore we did not respect the percentage of positive cells in the analysis. However, in other tumors we found a more heterogeneous distribution of staining. The cause could be the more homogeneous tumor differentiaton in cribriforme ACC compared e.g. with squamous cell carcinomas or glioblastomas $[7,8]$.

The expression of p-AKT was found as an independent parameter for higher relapse risk in ACC, apart from well documented predictors like incomplete surgical resection, which we also found correlated significantly with higher potential for relapsing. Activation and phosphorylation of the Akt/protein kinase B (AKT) by phosphatidylinositol 3kinase (PI3K) often appears in malignant tumors $[11,18]$. Phosphorylated AKT leads to increasing proliferation, tumor growth and decreasing apopotosis as well as stimulation of aerobic glycolysis in tumor cells. Elstrom et al. suggest that the activation of AKT could be responsible for the metabolic processes during the so-called Warburg effect $[8,11,18]$. One possible reason for AKT activation in ACC was shown by Hu et al., who found an expression of epiregulin (a member of epidermal growth factor family) in ACC cell lines with a higher metastatic risk [19]. 
Table 4: Multivariate analysis for relapse risk revealed expression of p-AKT and incomplete resection as prognostic parameters.

\begin{tabular}{|c|c|c|c|c|c|c|}
\hline \multirow[b]{2}{*}{ incomplete resection } & \multirow{2}{*}{\begin{tabular}{|l} 
beta \\
12.830
\end{tabular}} & \multirow{2}{*}{$\begin{array}{c}\text { SD }(\beta) \\
6.505\end{array}$} & \multirow{2}{*}{$\begin{array}{l}\mathbf{H R} \\
\max \end{array}$} & \multicolumn{2}{|c|}{$68 \%-\mathrm{Cl}$ von $\mathrm{HR}$} & \multirow{2}{*}{$\begin{array}{c}\text { P (Chi square) } \\
0.049\end{array}$} \\
\hline & & & & 558.725 & $\max$ & \\
\hline TKTL-I & 1.264 & 0.909 & 3.538 & 1.426 & 8.781 & 0.16 \\
\hline p-Akt & 4.858 & 2.394 & 128.739 & 11.754 & 1410.095 & 0.042 \\
\hline Glut-I & 1.100 & 0.880 & 3.003 & 1.245 & 7.244 & 0.21 \\
\hline M2PK & -5.252 & 2.818 & 0.005 & 0.000 & 0.088 & 0.062 \\
\hline incomplete resection & 9.811 & 5.436 & 18238.626 & 79.491 & $\max$ & 0.071 \\
\hline TKTL-I & 1.255 & 0.865 & 3.506 & 1.476 & 8.328 & 0.15 \\
\hline p-Akt & 4.188 & 2.162 & 65.911 & 7.588 & 572.488 & 0.053 \\
\hline M2PK & -4.400 & 2.508 & 0.012 & 0.001 & 0.151 & 0.079 \\
\hline incomplete resection & 5.962 & 2.814 & 388.324 & 23.289 & 6474.919 & 0.034 \\
\hline p-Akt & 2.662 & 1.167 & $|4.33|$ & 4.461 & 46.032 & 0.023 \\
\hline M2PK & -2.092 & 1.132 & 0.123 & 0.040 & 0.383 & 0.065 \\
\hline incomplete resection & 2.713 & 1.236 & 15.080 & 4.380 & 51.916 & 0.028 \\
\hline p-Akt & 1.109 & 0.529 & 3.032 & 1.787 & 5.143 & 0.036 \\
\hline
\end{tabular}

However, the expression of other parameters investigated in this study were not significantly associated with higher relapsing potential, despite the fact that they were found associated with worse clinical outcome in other malignant tumors.

TKTL-1 was postulated to be one of three isoforms of the thiamine-diphosphat dependent enzyme transketolase, which plays an important role in the pentose phosphate pathway (PPP) of anaerobic glycolysis [17]. This oxygenindependent pathway serves as an important source for the generation of $\mathrm{NADPH}_{2}$ needed in reductive biosynthesis (for example fatty acid synthesis), but more important is its role in nucleic acid ribose synthesis utilizing glucose carbons. More than $85 \%$ of ribose recovered from tumor cells is descended from this pathway. Therefore, transketolase upregulation in tumor progression seems very likely. We could show an expression of TKTL-1 in ACC as well as in some other malignant tumors $[7,8,10]$. However, the expression was not associated with relapse risk or survival. In other studies, tumors with an aggressive clinical course were investigated (e.g. glioblastomas [8]), whereas ACC grows comparatively slow. Only few ACC showd a strong expression of TKTL-1, more often the expression was only weak. With the background of the importance of the PPP in nucleic acid ribose synthesis, our findings fit with the slow growth of ACC. In accordance with the present results, we have not found a correlation between TKTL-1 expression and prognosis in tumors of lower malignancy like astrocytomas < grade IV or granulosa cell tumors of ovary $[8,9]$.

The non-predictive value of M2PK and GLUT-1 can be assumed in the same context. The glycolytic M2 pyruvate kinase isoenzyme (M2PK) plays a key role by channelling glucose carbons either into synthetic processes or towards glycolytic energy production, the former reactions being catalyzed by the dimeric form of $\mathrm{M} 2 \mathrm{PK}$, the latter by its tetrameric form. The dimeric form of $\mathrm{M} 2 \mathrm{PK}$ is characteristic for tumor cells [12] resulting in an accumulation of glycolytic metabolites, which are canalized into synthetic processes such as amino acid production or nucleic acid synthesis via the pentosephosphate cycle. Despite nonsignificant results, similar to other tumors a lower degree of M2PK expression tends to be an unfavorable parameter for prognosis. Interestingly, in our cohort elderly showed 
a lower expression, but this result was not found associated with another relapse risk in this subgroup. The expression and functionality of glucose transporters (GLUT) in tumors is probably related to a number of factors. In this context, the role of oncogenes and tumor hypoxia has generated a considerable interest [20]. In squamous cell carcinomas and other malignancies, hypoxia induces a higher expression of GLUT [21]. The expression of GLUT-1 was found in several malignant tumors, but not always in correlation with prognosis [9,13-15]. In our study, GLUT-1 was expressed in the majority of ACC, however the expression was mostly weak. Therefore we assume that hypoxia may play an minor role in progression of ACC. Further investigations regarding this point seems necessary (e.g. investigation of hypoxia inducible factor HIF- $1 \alpha$ and correlation with GLUT and a prospective study of GLUT function in ACC with 18-FDG-PET).

Due to the missing significance of TKTL-1, M2PK and GLUT-1, an influence of a glycolytic phenotype is not proved as parameter of the tumor progression of ACC. However, further studies with other markers seem necessary. Nevertheless, there was a correlation between expression of p-AKT and TKTL-1 as well as M2PK, so that the connection between these parameters reported in the literature seems once more plausible [8].

\section{Conclusion}

Expression of p-AKT is associated with a higher relapse risk in adenoid cystic carcinomas of salivary glands. In absence of other predictive parameters apart from tumor subtype and status of resection, the immunohistochemical staining could help to assess the individual risk for a more aggressive course of disease. Moreover, our observation is in particular important, hence investigations and clinical trials regarding the therapeutic target of the PI3KAKT pathway are ongoing [22,23].

\section{Competing interests}

The authors declare that they have no competing interests.

\section{Authors' contributions}

HUV: Main investigations, idea, and discussion; HKMH: Diagnoses; MaS, AB, AM: Clinical data, material, results; UK, MeS: Methodical approaches, theory, discussion; IH: Statistics. All authors read and approved the final manuscript.

\section{Acknowledgements}

We thank Michaela Kapp, Petra Stempfle, and Erwin Schmitt for excellent technical assistance.

\section{References}

I. Spiro RH, Huvos AG, Strong EW: Adenoid cystic carcinoma of salivary origin. A clinicopathologic study of 242 cases. Am J Surg 1974, 128:512-520.

2. Chummun S, McLean NR, Kelly CG, Dawes PJ, Meikle D, Fellows S, Soames JV: Adenoid cystic carcinoma of the head and neck. $\mathrm{Br}$ J Plast Surg 200I, 54:476-480.

3. Bradley PJ: Adenoid cystic carcinoma of the head and neck: a review. Curr Opin Otolaryngol Head Neck Surg 2004, I 2: I 27-I 32.

4. Dodd RL, Slevin NJ: Salivary gland adenoid cystic carcinoma: a review of chemotherapy and molecular therapies. Oral Oncol 2006, 42:759-769.

5. Chandana SR, Conley BA: Salivary gland cancers: current treatments, molecular characteristics and new therapies. Expert Rev Anticancer Ther 2008, 8:645-652.

6. Pfeffer MR, Talmi Y, Catane R, Symon Z, Yosepovitch A, Levitt M: A phase II study of Imatinib for advanced adenoid cystic carcinoma of head and neck salivary glands. Oral Oncol 2007, 43:33-36.

7. Volker HU, Scheich M, Schmausser B, Kammerer U, Eck M: Overexpression of transketolase TKTLI is associated with shorter survival in laryngeal squamous cell carcinomas. Eur Arch Otorhinolaryngol 2007, 264: |43|-|436.

8. Volker HU, Hagemann C, Coy J, Wittig R, Sommer S, Stojic J, Haubitz I, Vince GH, Kammerer U, Monoranu CM: Expression of transketolase-like I and activation of Akt in grade IV glioblastomas compared with grades II and III astrocytic gliomas. Am J Clin Pathol 2008, I30:50-57.

9. Schmidt M, Kammerer U, Segerer S, Cramer A, Kohrenhagen N, Dietl J, Voelker HU: Glucose metabolism and angiogenesis in granulosa cell tumors of the ovary: activation of Akt, expression of M2PK, TKTLI and VEGF. Eur J Obstet Gynecol Reprod Biol 2008, I39:72-78.

10. Kohrenhagen N, Voelker HU, Schmidt M, Kapp M, Krockenberger M, Frambach T, Dietl J, Kammerer U: Expression of transketolaselike I (TKTLI) and p-Akt correlates with the progression of cervical neoplasia. J Obstet Gynaecol Res 2008, 34:293-300.

II. Elstrom RL, Bauer DE, Buzzai M, Karnauskas R, Harris MH, Plas DR, Zhuang H, Cinalli RM, Alavi A, Rudin CM, Thompson CB: Akt stimulates aerobic glycolysis in cancer cells. Cancer Res 2004, 64:3892-3899.

12. Oremek GM, Teigelkamp S, Kramer W, Eigenbrodt E, Usadel KH: The pyruvate kinase isoenzyme tumor M2 (Tu M2-PK) as a tumor marker for renal carcinoma. Anticancer Res 1999, I9:2599-260I.

13. Kunkel M, Reichert TE, Benz P, Lehr HA, Jeong JH, Wieand S, Bartenstein P, Wagner W, Whiteside TL: Overexpression of Glut-I and increased glucose metabolism in tumors are associated with a poor prognosis in patients with oral squamous cell carcinoma. Cancer 2003, 97:1015-1024.

14. Kato Y, Tsuta K, Seki K, Maeshima AM, Watanabe S, Suzuki K, Asamura $H$, Tsuchiya $R$, Matsuno $Y$ : Immunohistochemical detection of GLUT-I can discriminate between reactive mesothelium and malignant mesothelioma. Mod Pathol 2007, 20:215-220.

15. Weiner MF, Miranda RN, Bardales RH, Mukunyadzi P, Baker SJ, Korourian S, De Las Casas LE: Diagnostic value of GLUT-I immunoreactivity to distinguish benign from malignant cystic squamous lesions of the head and neck in fine-needle aspiration biopsy material. Diagn Cytopathol 2004, 3 I :294-299.

16. Warburg O, Posener K, Negelein E: Über den Stoffwechsel der Carcinomzelle. Biochem Z 1924, I 52:309-344.

17. Coy JF, Dressler D, Wilde J, Schubert P: Mutations in the transketolase-like gene TKTLI: clinical implications for neurodegenerative diseases, diabetes and cancer. Clin Lab 2005, 5 I:257-273.

18. Schlegel J, Piontek G, Budde B, Neff F, Kraus A: The Akt/protein kinase $B$-dependent anti-apoptotic pathway and the mitogen-activated protein kinase cascade are alternatively activated in human glioblastoma multiforme. Cancer Lett 2000, I 58:103-108.

19. Hu K, Li SL, Gan YH, Wang CY, Yu GY: Epiregulin promotes migration and invasion of salivary adenoid cystic carcinoma cell line SACC-83 through activation of ERK and Akt. Oral Oncol 2009, 45:156-163. 
20. Dang CV, Lewis BC, Dolde C, Dang G, Shim H: Oncogenes in tumor metabolism, tumorigenesis, and apoptosis. J Bioenerg Biomembr 1997, 29:345-354.

21. Airley R, Loncaster J, Davidson S, Bromley M, Roberts S, Patterson A, Hunter R, Stratford I, West C: Glucose transporter glut-I expression correlates with tumor hypoxia and predicts metastasis-free survival in advanced carcinoma of the cervix. Clin Cancer Res 200I, 7:928-934.

22. Yap TA, Garrett MD, Walton MI, Raynaud F, de Bono JS, Workman P: Targeting the PI3K-AKT-mTOR pathway: progress, pitfalls, and promises. Curr Opin Pharmacol 2008, 8:393-4I2.

23. Koul D, Shen R, Bergh S, Lu Y, de Groot JF, Liu TJ, Mills GB, Yung WK: Targeting integrin-linked kinase inhibits Akt signaling pathways and decreases tumor progression of human glioblastoma. Mol Cancer Ther 2005, 4: I68I-1688.

Publish with Bio Med Central and every scientist can read your work free of charge

"BioMed Central will be the most significant development for disseminating the results of biomedical research in our lifetime. "

Sir Paul Nurse, Cancer Research UK

Your research papers will be:

- available free of charge to the entire biomedical community

- peer reviewed and published immediately upon acceptance

- cited in PubMed and archived on PubMed Central

- yours - you keep the copyright

Submit your manuscript here:

http://www.biomedcentral.com/info/publishing_adv.asp 